\title{
Uncertainty Quantification of petrophysical parameters obtained from well logging and core data from carbonate formation using Monte Carlo simulation and fuzzy logic.
}

Francioli, A.; Carrasco, A. UFF/TEQ - Niterói, RJ

Copyright 2019, SBGf - Sociedade Brasileira de Geofísica

This paper was prepared for presentation during the $16^{\text {th }}$ International Congress of the Brazilian Geophysical Society held in Rio de Janeiro, Brazil, August 19-22, 2019.

Contents of this paper were reviewed by the Technical Committee of the $16^{\text {th }}$ International Congress of the Brazilian Geophysical Society and do not necessarily represent any position of the SBGf, its officers or members. Electronic reproduction or storage of any part of this paper for commercial purposes without the written consent of the Brazilian Geophysical Society is prohibited.

\section{Abstract}

Information coming from petrophysical parameters is of great importance in reservoir engineering and for reserves evaluation. Rock petrophysics measurements involves some degree of uncertainties especially when carbonates formations are present, because they are characterized for their variation in porosity systems, such as intercrystalline, intergrain, moldic, vuggy and fracture. Porous system in carbonate formation are more complex than clastic formation, because carbonates are a mixture of these porous systems. The quantitative porosity and permeability values are difficult to predict and logic fuzzy was used to obtain the permeability, taken some data core as reference. Finally, the use of Monte Carlo technique is a helpful tool to quantify these uncertainties. In order to make this work, we use some well logging and data core in order to estimate probability distributions of this parameter.

\section{Introduction}

Theoretical models based on the physics of rocks sets relationships between the porous structure and the elastic, radioactive and electrical properties. Although significant progress has happened in modeling problems about porous structure obtained from physical properties of rock, factors as multiplicity of responses, computational stability and availability of core analysis information, limits their reliable petrophysical characterization (Wu \& Chen, 2014).

Carbonate formation has complex porosity and permeability. To establish a classification of the carbonates porosities, Choquette - Pray in 1970 recognized the need to include the period and their origin in the description. They considered 15 types of pores arranged in three classes dependent on the fabric. The porosity with selective fabric can be depositional, diagenetic or both of them. No selective fabrics include fractures or cavities formed by dissolution process. Fabric selective or not, is a category that includes animal or plants characteristics or from erosion or tectonic process (Ahr, 2008). Lucia (1995), established another classification in 1983, making a division between two types of porosities: intergrain (Figure 1) and moldic (isolated or connected by microfractures). This classifica- tion also has a division, since the intergrain porosity would have a fabric mainly with grains (Figure $1 \mathrm{~A}$ ) or having a mud fabric. Likewise, the vuggy porosity without connection can be moldic (Figure 1B), intrafossil or intragrain. The subdivision for the connected vugular porosity can be: cave type, fracture, fractures extended by dissolving processes, fenestral, or moldic with microfractures interconnection (Figure 1C), (Lucia, 2004).
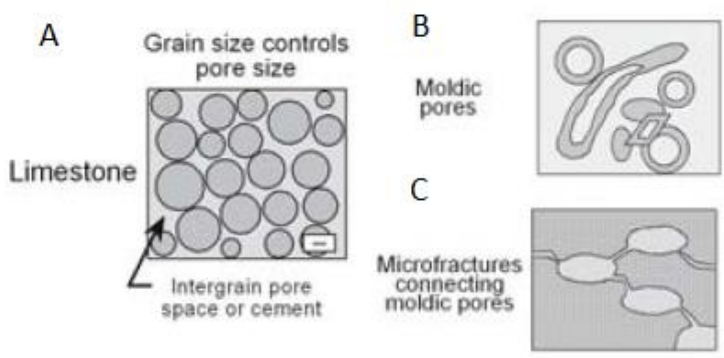

Figure 1. Porosity classification by Lucia (modified Lucia, 2004)

This complexity in porosities has a direct effect in the permeability, and for making prediction of these values, the application of logic fuzzy in data well logging is an important tool.

Fuzzy logic is an extension of conventional Boolean logic (zeros and ones) developed to handle the concept of partial truth - truth values between 'completely true' and 'completely false'. It was introduced by Dr. Lotfi Zadeh of UC/Berkeley in the 1960's as a means to model uncertainty (Cuddy, 1997). Fuzzy logic was applied to lithofacies and permeability forecasting by Hambalek and González (2003) using well logging in eastern Venezuela for eighteen wells of the same area having only electrical logs. In 2008, Ghafoori and Sajjadian studied a permeability in Iranian carbonate reservoir for predicting these values using Fuzzy Logic obtaining important results, but involving some level of uncertainty.

Use of Monte Carlo technique to quantify uncertainties dates as early as 1964, where the technique was applied to business decisions. Since then, Monte Carlo has been used to help simulate the economic model for exploration prospects, to estimate risk and manage performance associated with drilling and to estimate reservoir properties (Varghese \& Kumar, 2005).

Monte Carlo processing is used to assess the uncertainties in many different fields of measurement and analysis. Sensitivity analysis in Monte Carlo processing allows the interpreter to assess the relative impact of each input 
uncertainty. This can lead to an assessment of the value of the information which is required to eliminate the larger uncertainties. (Aldred, 2018). Some petrophysical deliverables for volumetric purposes are porosity, permeability and water saturation. These data are usually provided without quantitative determination of their uncertainties and most of them are critical values for modeling purposes (Adams, 2005). This work will use the permeability result from logic Fuzzy application on well logging data to illustrate the relationship between uncertainty and responses obtained in carbonates formation.

\section{Method}

The studied field is an heterogeneous carbonate reservoir located in the southwest of Brazil. The lithology of the reservoir is limestone. Permeability in carbonate formations is not only related to porosity, but it also depends on grain size, sorting, pore throat sizes of inter-granular pore space, the amount of unconsolidated vugs (fratures and solution cavities) an the presence or absence of connected vugs as discussed by Lucia (2004).

For this reason, the data for this study is composed by a set of openhole logs for an interval of $5580-5820 \mathrm{~m}$ and a group of 81 permeability core data as shown in track 6 of Figure 2.

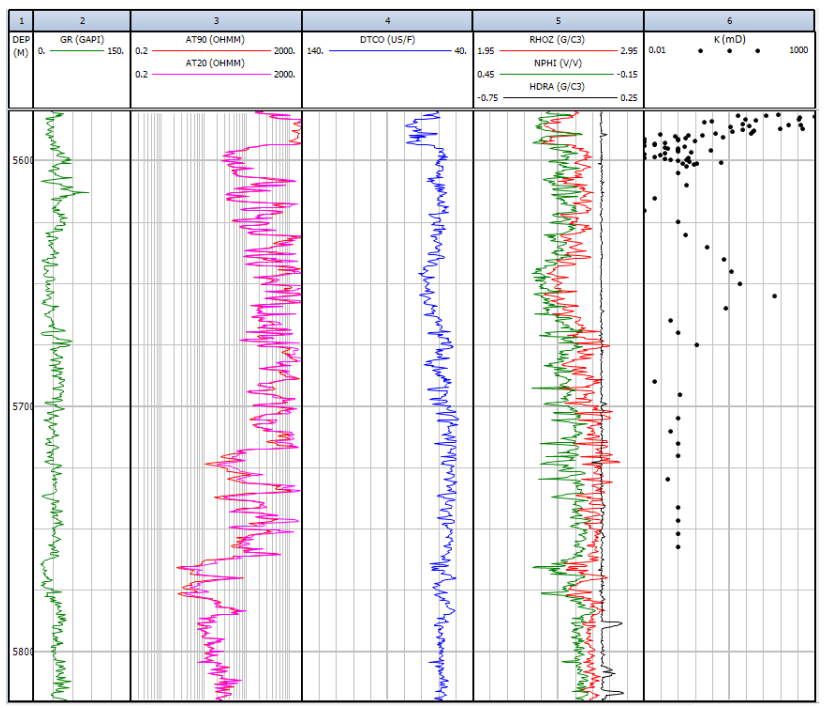

Figure 2. Input well logging and core data

The group of input well logging data consists of gamma ray (GR, track 2 ), deep resistivity log (AIT90) and shallow resistivity log (AIT20) (track 3 ) showing high values in $5580-5750 \mathrm{~m}$, and sonic log (DTCO) on track4. Density (RHOZ, HDRA) and neutron (NPHI) logs are displayed in track 5 , and permeability $(\mathrm{mD})$ data core on track 6 . Most of core data was taken from the hydrocarbon bearing layer, with most of this information located in the upper part of the log.

Initially, a preliminary interpretation was made using the open hole mentioned as input data in order to evaluate the radioactivity level of the carbonate formation and saturation results. For analyses of radioactivity level was used gamma ray and density joined with neutron logs, to obtain the total porosity.

For application of fuzzy logic, core data permeability may be classified as excellent, good and poor with respect to the cutoff values. Using fuzzy sets, each value will be a member of each fuzzy set to a membership degree. For permeability prediction, the interval core permeability data and the well logging data was loaded into the IP (Interactive Petrophysics) software. For this case study uses the density, neutron, sonic, resistivity and gamma ray data. The program first sorts the permeability data in an increasing order and then the other input curves data will be assigned according to their permeability values in each bin. Different bin numbers can be assigned and the result will be dependent on bin numbers. The number of bins depends on the number and the range of the input data (Ghafoori \& Sajjadian, 2008). The vertical green lines represent one standard deviation either side of the mean, as shown in Figure 3. In the first graphic of permeability $(\mathrm{K})$, core data is not continuing.
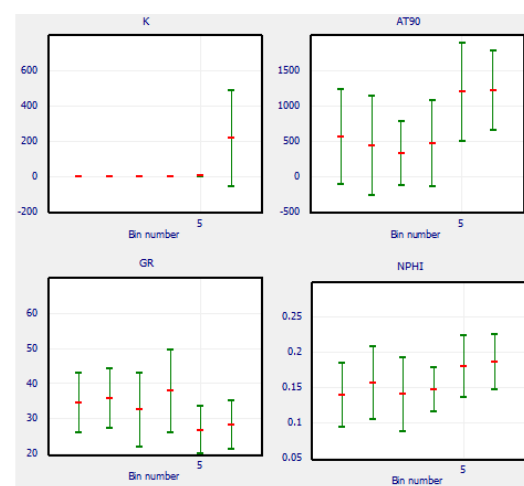

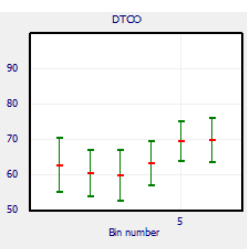

$\mathrm{RHOZ}$

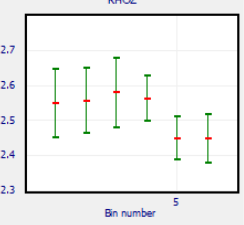

Figure 3. Curve bin distribution cross plot for fuzzy logic

To make the prediction, the program first calculates the fuzzy probability that an input log is in a certain bin. The following equation is used for this:

$$
P\left(C_{b}\right)=\sqrt{n_{b}} \times e^{\frac{-\left(C-\mu_{b}\right)^{2}}{2 \sigma_{b}^{2}}} \text { (eq.1) }
$$

Where $P\left(C_{b}\right)$ is the probability that curve $C$ is in bin ' $b$ ', $n_{b}$ is the number of samples in bin ' $b$ ', $C$ is the input values for curve $\mathrm{C}, \mu_{b}$ is the mean value for curve $\mathrm{C}$ form bin ' $\mathrm{b}$ ', and $\sigma_{b}$ is the standard deviation for curve $\mathrm{C}$ for bin ' $\mathrm{b}$ '.

The probabilities for all the input curves are then combined as follows: $s$

$$
\frac{1}{P_{b}}=\frac{1}{P\left(C 1_{b}\right)}+\frac{1}{P\left(C 2_{b}\right)}+\frac{1}{P\left(C 3_{b}\right)}+\ldots+\frac{1}{P\left(C n_{b}\right)} \text { (eq.2) }
$$

Where $P_{b}$ is the probability for bin ' $b$ ', and $P\left(C n_{b}\right)$ is the probability for curve Cn for bin 'b'. The most likely solution will be the bin with the highest probability (Senergy, 2014).

To estimate the error in the petrophysical analysis, Monte Carlo simulation is applied using the Gaussian distribution. This involved mainly calculation of radioactivity level, porosity and saturation determination and finally summing up for the fuzzy responses. Calculating some 
petrophysical logs performs simulation analysis. A reciprocal shift is then applied to various parameter used and finally a ramdom Gaussian distribution is applied.

\section{Results}

In the upper part of resistivity log (track 3 of Figure 2), the high values registered corresponds to hydrocarbon layer and most of the core data correspond to the upper part of this carbonate layer. Permeability values vary from 0.01 to $1000 \mathrm{mD}$ as can be observed on track 6 of Figure 2 and there is no relation with its lower part. After applying Fuzzy the results obtained is in Figure 4.

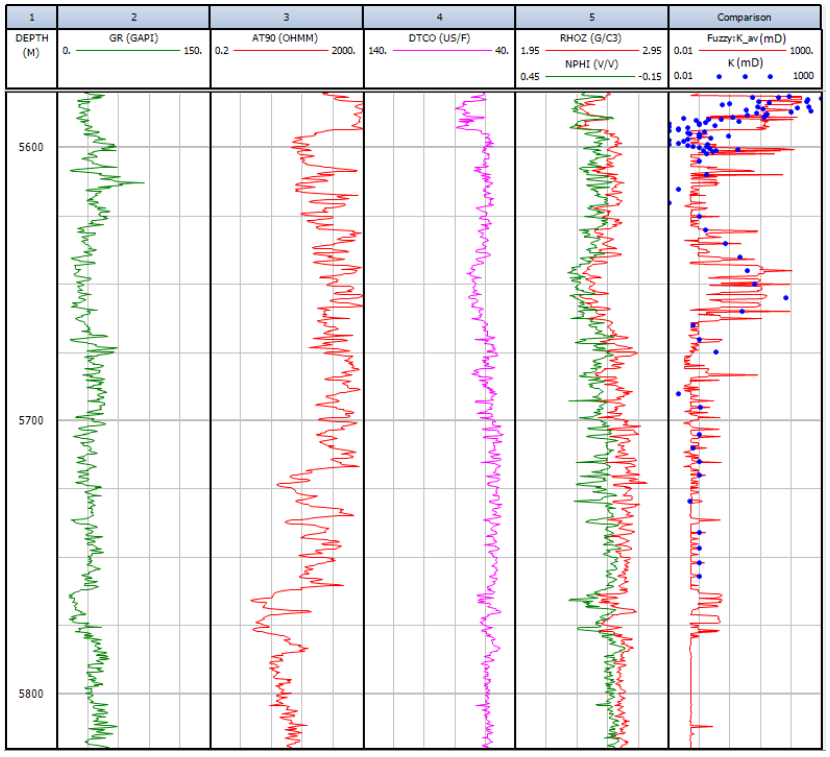

Figure 4. Comparison of predicted permeability from fuzzy logic and core data.

This figure shows the input data used for the application of fuzzy logic: gamma ray (track 2), resistivity (track 3 ), sonic (track 4), density and neutron (track 5), and core data (track 6). In this last track shows the predicted permeability from fuzzy logic (red curve) together with the real core data (blue dots) and it is possible to note that there is some coherence between both values. The comparison of permeability values reveals little difference in the permeability values. The upper part of the predicted curve shows a great variation in the permeability values and this information is support by the core data, and in the lower part there are some few and low values of permeability, which is consistent with core data.

Finally Monte Carlo simulation is applied taking fuzzy results permeability in order to quantify uncertainties. Figure 5 shows a histogram of the permeability values through various pay layers after 500 iterations. Figure 6 shows a summary result which gives the output in various percentiles values: P10, P50 and P90. When the range of uncertainty is represented by a probability distribution, a low, best and high estimate shall be provide by these terms. $\mathrm{P} 10$ is a $10 \%$ probability that the values predicted will equal or exceed the high estimate, P50 is a $50 \%$ probability that the values found will equal or exceed the best estimate and P90 is a $90 \%$ probability that the values will equal or exceed the low estimate.

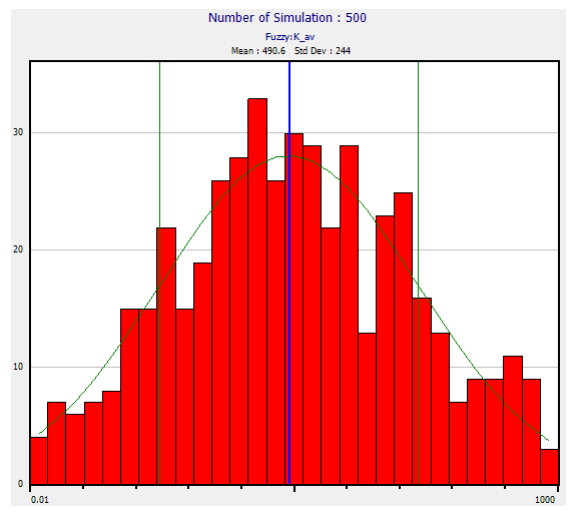

Figure 5. Histogram of Permeability values using Monte Carlo Simulation after 500 iterations.

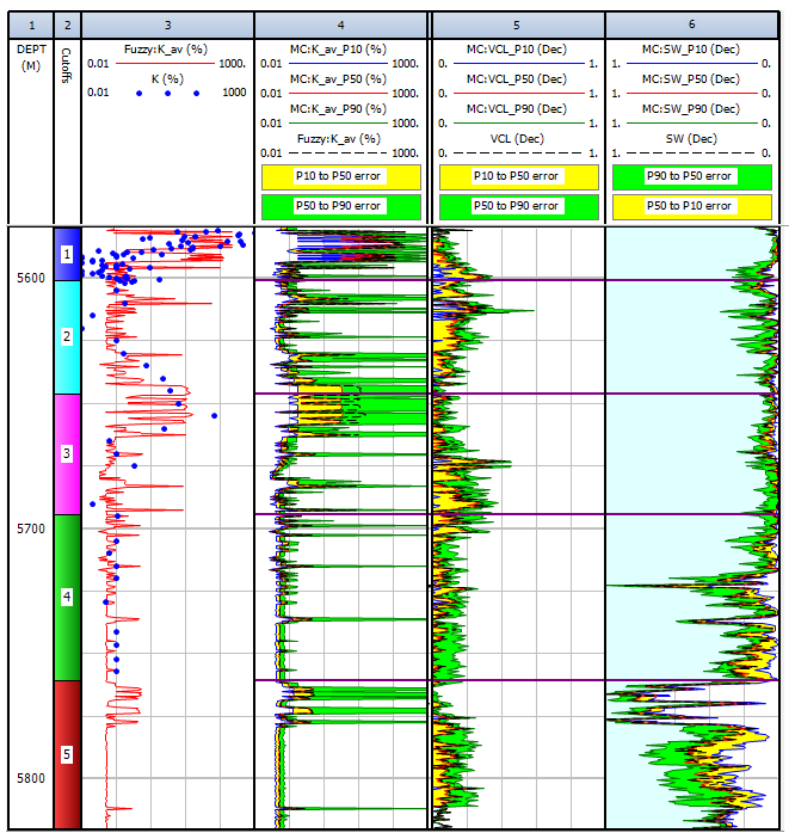

Figure 6. Monte Carlo simulation results

In the first track of Figure 6, the depth of the records were obtained in meters, the second track shows the depth separation in 5 zones, based on a preliminary interpretation, considering the electric log (AT90), radioactive (GR), porosities (RHOZ and NPHI) and permeability values of the core data. Thus, in zone 1 can be observed erratic permeability values, zone 2 and 3 characterized by increasing and decreasing permeability values, zone 4 by constant values and zone 5 , in absence of data core. In track 3 we have the values of the permeability from core (blue dots) and the curve in red is the result of the application of the fuzzy logic. The next tracks were obtained by the application of the Monte Carlo technique in the results obtained by logic Fuzzy and radioactivity and saturations calculations. So, track 4 shows the application of the Monte Carlo technique as a function of the P10, P50 and 
P90 probability parameters on the Fuzzy responses, noting that in zone 1 and at the top of zone 2 there is little reliability in the results obtained, different from zone 2, 4 and 5. In track 5, the Monte Carlo technique was applied on the radioactivity logs and indicate that the best answers correspond to the lowest values of radioactivity (yellow area) and finally in track 6, Monte Carlo application in water saturation calculation (using the Simandoux) relationship), results in high water saturation values at the lower part of the track (lower zone 4 and zone 5).

\section{Conclusions}

Carbonate formations are characterized by their complexity as the measurement of the petrophysical parameters of porosity and permeability, since it is known that carbonates have several types of porosity, because their origin depends on diagenetic, chemical and tectonic processes. The use of fuzzy logic has proved to be an adequate tool for the study of complex data and it is an application of recognized statistical technique. Permeability prediction for this complex formation results in good fit, taking into consideration the core data as a reference.

In order to evaluate the fuzzy prediction, we use the Monte Carlo technique, given important results in choosing the best estimates for each zone for permeability data and saturation calculation. It is still possible for calculated uncertainty ranges without using Fuzzy logic and using other validation mechanisms.

\section{Acknowledgement}

To the Federal Fluminense University (UFF) by infraesstructure, LENEP - UENF, and Senergy Softwares by the academic license of Interactive Petrophysics (IP).

\section{References}

Adams, S. J. Quantify Petrophysical Uncertainties. SPE 93125. 2005.

Aldred, Rick. Monte Carlo Processing of Petrophysical Uncertainty. Annual Logging Symposium. SPWLA. 2018.

Cuddy, Steve. The Application of the Mathematics of Fuzzy Logic to Petrophysics. 1997.

Ghafoori, M. R. \& Sajjadian. A state-of-the-art Permeability Modeling using Fuzzy Logic in a Heterogeneous Carbonate (an Iranian Carbonate Reservoir Case Study). IPTC 12019. 2008.

Hambalek, Nancy \& González, Reinaldo. Fuzzy Logic applied to lithofacies and permeability forecasting. SPE 81078. 2003.

Kumar, Bishnu \& Varghese, José. Application of Monte Carlo Simulation to Quantify Uncertainties In Petrophisical Deliverables. Geohorizons, July, 2005.

Lucia, F.J. Carbonate Reservoir Characterization - An Integrated Approach, $2^{\text {nd }}$ edition, 336p. 2004.
Senergy. Interactive Petrophysics. User Manual. 2014.

Wu, Baohai \& Chen, Liping. 2014. Optimizing calculation of multiple porosities in carbonates with petrophysics and rock physics integration strategy. SEG-2014-0906. 